\title{
Computationally constructing a repository of compound remote associates test items in American English with comRAT-G
}

\author{
Ana-Maria Olteţeanu ${ }^{1}$ (D) - Holger Schultheis ${ }^{1}$ (i) - Jonathan B. Dyer ${ }^{2}$ (i)
}

Published online: 12 December 2017

(C) Psychonomic Society, Inc. 2017

\begin{abstract}
The Remote Associates Test (RAT) has been used to measure creativity, however few repositories or standardizations of test items exist, like the normative data on 144 items provided by Bowden and Jung-Beeman. comRAT is a computational solver which has been used to solve the compound RAT in linguistic and visual forms, showing correlation to human performance over the normative data provided by Bowden and Jung-Beeman. This paper describes using a variant of comRAT, comRAT-G, to generate and construct a repository of compound RAT items for use in the cognitive psychology and cognitive modeling community. Around 17 million compound Remote Associates Test items are created from nouns alone, aiming to provide control over (i) frequency of occurrence of query items, (ii) answer items, (iii) the probability of coming up with an answer, (iv) keeping one or more query items constant and (v) keeping the answer constant. Queries produced by comRAT-G are evaluated in a study in comparison with queries from the normative dataset of Bowden and JungBeeman, showing that comRAT-G queries are similar to the established query set.
\end{abstract}

Keywords Remote associates test - Creative cognition . Computational creativity $\cdot$ Cognitive modeling

Ana-Maria Olteţeanu

amoodu@informatik.uni-bremen.de

1 Cognitive Systems, Bremen Spatial Cognition Center, University of Bremen, Enrique-Schmidt-Straße 5, 28359 Bremen, Germany

2 Department of Computer Science, University of Pittsburgh, 4200 Fifth Ave, Pittsburgh, PA 15260, USA

\section{Introduction}

Imagine you set out to test or manipulate the creative ability of some of your participants using the Remote Associates Test (Mednick \& Mednick, 1971). This test consists of giving participants three words-like WATER, MINE, and SHAKER - and asking them what word might be related to all three such words. ${ }^{1}$ You would have to first find and select a set of RAT queries, and then control as many variables as you can about them.

Various creativity tests are available: the Alternative Uses Test (Guilford, 1967), the Remote Associates Test (Mednick \& Mednick, 1971), the Torrance Tests of Creative Thinking (Kim, 2006), the Wallach-Kogan tests (Wallach \& Kogan, 1965), riddles (used by Whitt \& Prentice, 1977; Qiu et al., 2008), empirical insight tests from Duncker (1945), Maier (1931), and Saugstad and Raaheim (1957) and others. However, some of these tests are not easily available (TTCT), some are non-standardized or do not provide any norms, and others provide small sets of stimuli. Thus, various creativity tests could highly benefit from modernization by being normed, having more factors controlled for and by developing ampler sets of stimuli, in order to provide more varied testing conditions.

The Remote Associates Test (Mednick \& Mednick, 1971), has been used to measure creativity and adapted to various other languages - for example in Japanese (Baba, 1982) and Dutch (Chermahini, Hickendorff, \& Hommel, 2012). The test has been rated as the second most used creativity test in a meta-analysis surveying 45 neuroimaging studies (Arden, Chavez, Grazioplene, \& Jung, 2010). The RAT is assumed to measure creative convergent thinking,

\footnotetext{
${ }^{1}$ The answer to this RAT query is SALT.
} 
unlike other creativity tests, which are better suited to measure divergent thinking-e.g., the Alternative Uses Test. The RAT is very useful in measuring insight theory effects, as performance in the RAT has been shown to correlate with performance in insight problems (Schooler \& Melcher, 1995).

Various types of investigations in and beyond creative cognition use the Remote Associates Test. Amongst others, these include the study of the effects of incubation with GO players (Sio \& Rudowicz, 2007) and baseball players (Wiley, 1998), the relation between REM sleep and creativity (Cai, Mednick, Harrison, Kanady, \& Mednick, 2009), synesthesia and creativity (Sitton \& Pierce, 2004; Ward, Thompson-Lake, Ely, \& Kaminski, 2008), the role of affect in problem-solving (Fodor, 1999), memory (Storm, Angello, \& Bjork, 2011), peripheral attention (Ansburg $\&$ Hill, 2003) etc. Because of its wide use, the scientific community would thus benefit from an ampler set of standardized stimuli for the RAT.

Worthen and Clark (1971) made the case that different stimuli categories can be distinguished within the original stimuli of Mednick and Mednick (1971)—specifically they differentiated between functional items and structural items (sometimes also appearing in the literature under the name of compound items).

The CreaCogs framework (Olteţeanu, 2014, 2016) for creative problem-solving uses knowledge organization to support a unified set of core creative problem solving processes, like association, associative convergence, re-representation, restructuring, search, and substitution. The validation of the framework and processes is done in a comparative manner to human performance (Olteţeanu, Falomir, \& Freksa, in press) through implementing systems which can show creative problemsolving abilities and solve creativity tests for humans. Among such systems, the comRAT-C system (Olteţeanu \& Falomir, 2015) has explicitly addressed the computational solving of compound RAT queries. When solving the RAT, comRAT-C calculated the probability of finding a solution based on the frequency of query and answer words, as will be shown in "Generating new remote associates test items with comRAT-G". A highly significant correlation has been observed between the results of comRAT-C and the difficulty of RAT queries for humans, as expressed in percentage of solvers and response times in the human normative data (Bowden \& Jung-Beeman, 2003). This correlation ranged between 0.3 and 0.52 for different solving times. This correlation showed that the frequency of query items plays an important role into how difficult a query is for human participants, and a standardized set of RAT stimuli should take this into account. Furthermore,
(Oltețeanu \& Schultheis, in press) modified frequency and probability factors independently, keeping them at low and high levels. This showed that frequency and probability both are factors which influence accuracy and response times when solving the RAT.

For the Remote Associates Test, normative data from human participants does exist from Bowden and JungBeeman (2003), which provides data on mean time-tosolution and percentage of participants solving for 144 compound RAT problems, with four different time limits. Though very useful, this work does not provide standardized queries based on frequency of occurrence of word or answer stimuli, the importance of which has been shown by Olteţeanu and Falomir (2015). This paper aims to enrich the existing pool of compound Remote Associates Test items and provide a standardized treatment that allows control over the frequency of occurrence and probability of finding an answer. Seventeen million new compound RAT items are constructed, using the entire space of frequent noun expressions in American English - thus providing the largest standardized treatment of the compound RAT test to date. These items are computationally generated by adapting the previously implemented computational solver of the Remote Associates Test, comRAT-C (Olteţeanu \& Falomir, 2015), to a generative variant - comRAT-G. The frequency of items from the COCA corpus ${ }^{2}$ and comRAT-C's probability of finding an answer are indexed in the provided repository, and can be used to generate a set of controlled queries. Subsets of queries in which one word or the answer are kept constant can also be extracted.

The rest of this paper is structured as follows. A brief overview of the comRAT solver and its transformation to comRAT-G is provided in "From comRAT to comRAT-G". The methodology of generating new Remote Associates Test items with comRAT-G is explained, together with examples, in "Generating new remote associates test items with comRAT-G". The evaluation of 100 queries with human participants is shown in "Evaluation". The type of data generated in the repository is described in "The Repository", and various possible uses are showcased. Finally, future work is discussed. The 100 items used for evaluation are presented in the Annex.

\section{From comRAT to comRAT-G}

The comRAT-C (Olteţeanu \& Falomir, 2015) system has been built to solve the compound variant of the

\footnotetext{
${ }^{2}$ Corpus of Contemporary American English (COCA): http://corpus. byu.edu/coca/
} 


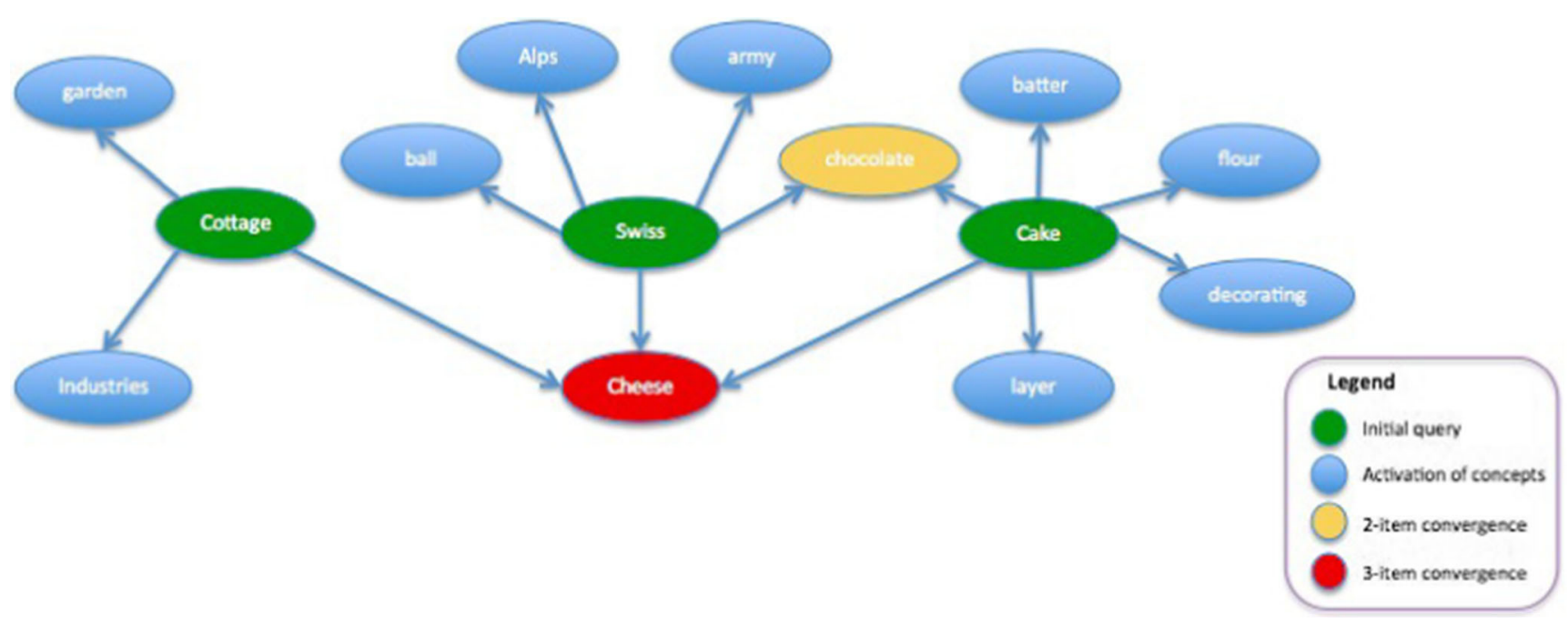

Fig. 1 A graphical depiction of the link structure obtained in comRAT-C. Only a few links are shown for visual readability

Remote Associates Test, in the tradition of Psychometric AI (Bringsjord, 2011) and as an exploration of the processes of the CreaCogs creative problem-solving framework (Olteţeanu, 2014; Olteţeanu \& Falomir, 2016).

As data, comRAT-C takes the most frequent 2-grams from the COCA corpus. Knowledge organization plays an important role in CreaCogs and in comRAT's knowledge base in the following way: whenever a 2-gram is given to comRAT-C, it is stored as an object of the Expression class, which is constructed from two objects of the Concept class and a Link between them. If one of the Concepts is already present, the other Concept and the new Link are added. If both Concepts are present, only the Link is added. For example, if the 2-gram "cake flour" is read, comRAT-C will check to see if it knows the Concepts "cake" and "flour". If it doesn't know one of them, the item will be added as a Concept ${ }^{3}$; if it doesn't know either, both items will be added. Then it will add a link between those Concepts, with a numeric tag attached-the frequency of the 2-gram as taken from the corpus.

Over time, each Concept will end up with a set of Links to all the other Concepts it has been in an Expression with, as shown in Fig. 1. This associative structure constitutes the knowledge organization of comRAT-C. When three words are given to comRAT-C, as they would in the context of a RAT query, each of these words activates the set of Concepts

\footnotetext{
${ }^{3}$ The reason we call these items Concepts is that we entertain the possibility that they can be more than linguistic items, and another system - comRAT-V (Olteţeanu, Gautam, \& Falomir, 2015)- aims to solve a visual variant of the RAT using visual associations.
}

they are linked to. An overlapping activation starting from two or three of the initial words can sometimes be observed. In Fig. 1, the initial words are depicted in green - COTTAGE, SWISS, and CHEESE. A two-item convergence of activation is observed for the word CHOCOLATE, and a three-item convergence for the word CHEESE. The three-item convergences are possible RAT query responses. Multiple two and three-item convergences are of course possible for the same query.

The comRAT-C system performs well in answering compound RAT queries even without the links between Concepts being weighted using the frequency of 2-gram metrics; however, adding these metrics improves comRAT-C's performance and helps break ties between multiple possible three-item convergences.

An analysis of comRAT-C's probability to find an answer based on the two-gram frequency data revealed a correlation to human performance normative data. This correlation shows that the frequency of 2-grams, on which the probability of finding the answer is based, might have an influence on the process of solving compound RAT queries. In order to keep compound queries controlled for the frequency variable, to check for other influences (like order), and to understand these influences in more detail, frequencybased probability data needs to be gathered on a large set of queries. A large enough set of queries can also be used to keep part of the query words or the answer word (different queries, same answer) constant. In order to gather such data, and construct a large set of queries, we proposed reverse-engineering our computational approach in order to generate new compound RAT items. 
Table 1 An example of preliminary results, focusing on words as answers to possible queries

\begin{tabular}{lllll}
\hline$w_{\text {ans }}$ & $w_{q}$ & $f r\left(w_{\text {ans }}, w_{q}\right)$ & $\sum_{k=1}^{m}\left(w_{q}, w_{k}\right)$ & $P\left(w_{\text {ans }} \mid w_{q}\right)$ \\
\hline Health & animal & 95 & 6558 & 0.0145 \\
& bone & 159 & 4048 & 0.0393 \\
& brain & 25 & 7139 & 0.0035 \\
& breast & 46 & 10302 & 0.0045 \\
& child & 149 & 16550 & 0.009 \\
& city & 49 & 16666 & 0.0029 \\
\hline
\end{tabular}

In this case the answer word is "health", and only a few of its 256 links are shown

Thus, instead of using the organization structure of comRAT to provide answers, this will be used to provide queries. From this vantage point, each word $w_{\text {ans }}$ that has at least three links, let's say to words $w_{a}, w_{b}, w_{c}$, is a potential answer to a RAT query.

\section{Generating new remote associates test items with comRAT-G}

The process of generating new Remote Associates Test items unfolds as follows. All the noun-noun high frequency 2-grams in COCA are organized in Concepts and Links in the knowledge base of comRAT-G. The selection of noun-noun 2-grams is done using the UCREL CLAWS7 Tagset $^{4}$ (tags as per this tagset are provided with the 2-grams dataset). comRAT-G uses nouns alone in this current version, unlike comRAT-C, which used more parts of speech, as described in Olteţeanu and Falomir (2015). Thus the set of most frequent 2 million 2-grams is reduced to a set of 43,908 expressions.

First, comRAT-G iterates through the words and provides preliminary results, which henceforth we shall call type 1 results, a sample of which is shown in Table 1. Type 1 results consider each word as a potential answer word. Thus in Table 1, $w_{\text {ans }}$ stands for the answer word and $w_{q}$ for a potential query word which can be used to get the answer word. Terms $w_{q}$ can be further integrated in a RAT query in positions $w_{a}, w_{b}$ or $w_{c}$. The third column represents the frequency of association between the query word $w_{q}$ and the answer word $w_{\text {ans }}$. The fourth column represents the frequency of association between the query word and any word. The fifth column represents the

\footnotetext{
${ }^{4}$ http://ucrel.lancs.ac.uk/claws7tags.html
}

probability of answer $w_{\text {ans }}$ given specific query word $w_{q}{ }^{5}$ This is calculated as shown in Eq. 1. Query words $w_{q}$ are only generated for the $w_{a n s}$, which have at least three $w_{q}$. Applying the process to get to type 1 results yields a total of $\sim 81500$ unique $w_{a n s}, w_{q}$ combinations, based on 9601 unique answer words.

$$
P\left[w_{\text {ans }} \mid w_{q}\right]=\frac{f r\left(w_{q} w_{\text {ans }}\right)}{\sum_{k=1}^{n}\left(w_{q}, w_{k}\right)}
$$

After type 1 results have been produced and stored, the new RAT queries are generated, using a combinatoric approach. For each answer word, the set of query words are retrieved and three-word combinations are generated. This applies the well-known combinatorics formula shown in Eq. 2, with $n$ being the number of query items connected to a specific answer word, and $k$ being 3 .

${ }_{k}^{n}=\frac{n !}{k !(n-k) !}$

For each possible answer with $n<100$ (and of course $n>2$ ), all unique combination triples are produced. We capped $n$ at 100 because of computational costs $\left(\left(\begin{array}{c}100 \\ 3\end{array}\right)\right.$ is 161,700 possible combinations with the terms connected to the same answer word) and diminishing returns - an answer word $w_{\text {ans }}$ connected to over 100 items might be a very common word, or form much weaker bonds with either of the words; thus RAT items constructed from its terms might not be too interesting or intuitive to solve (lower associative power of triggering result).

In order to construct all such combinations in a computationally feasible manner, comRAT-G uses Alan Tucker's combinatorics algorithm (Tucker, 2006). For 9601 query answer words and capping $n$ at 100 (which translates into only using about 9200 answers), we obtain about 17 million possible RAT triples. The probability of answering the query is calculated based on the conditional probability of the answer to be triggered by each of the three query items. The probability thus currently considers an equal weighting of the three items, as shown in Eq. 3 (as in comRAT-C (Olteţeanu \& Falomir, 2015)). However, different weighting schemes can be considered for modeling purposes-which is why we also provide the conditional probability of each

\footnotetext{
${ }^{5}$ Further restrictions would have been possible, for example, by only considering words as valid query words, if their conditional probability is above a certain threshold. While this may have restricted possible query words to those that have a more valid/reasonable appeal, we thought it better to start with an all-inclusive set, on which then modelers can put their own thresholds.
} 
item, as shown in the Appendix. The various types of data items captured by this ample list of possible RAT queries and the roles in which such data can be used in empirical research are presented in "The Repository".

$P\left(w_{\text {ans }}\right)=\frac{P\left(w_{\text {ans }} \mid w_{a}\right)+P\left(w_{\text {ans }} \mid w_{b}\right)+P\left(w_{\text {ans }} \mid w_{c}\right)}{3}$

\section{Evaluation}

In order to check whether the queries created by comRAT$\mathrm{G}$ are suitable, valid and reliable RAT queries, which can be solved by human participants and are coherent with existing RAT datasets, we have set up a study in which the human performance on comRAT-G queries is compared to that on normative data from Bowden and Jung-Beeman (2003).

\section{Method}

Two sets of query items, one comprising randomly selected comRAT-G queries and the second comprising randomly selected queries from the normative data of Bowden and Jung-Beeman (2003), were presented mixed in random

Table 2 Descriptive data on the age, education, and self-rated creativity level of the participants, $n=113$

\begin{tabular}{lll}
\hline Indicator & Level & No. of participants \\
\hline Age & Under 20 & 2 \\
& $20-30$ & 33 \\
& $30-40$ & 36 \\
& $40-50$ & 17 \\
& $50-60$ & 20 \\
& $60-70$ & 4 \\
Education & Over 70 & 1 \\
level & High school diploma & 7 \\
& Enrolled in undergraduate courses & 27 \\
& Completed undergraduate courses & 34 \\
& Enrolled in postgraduate courses & 6 \\
& Completed postgraduate courses & 19 \\
& & 5 \\
Creativity & Low & 39 \\
self-rated & Average & 41 \\
& Above average & 22 \\
& High & 6 \\
& Very high & \\
& & \\
& &
\end{tabular}

Table 3 Descriptive statistics on accuracy in number of queries solved, $n=113$

\begin{tabular}{lllll}
\hline & $\begin{array}{l}\text { Mean accuracy } \\
\text { no. solved }(S D)\end{array}$ & $\begin{array}{l}\text { Std. } \\
\text { Error }\end{array}$ & $\begin{array}{l}95 \% \text { Conf. } \\
\text { L.B. }\end{array}$ & $\begin{array}{l}\text { interval } \\
\text { U.B. }\end{array}$ \\
\hline comRAT-G & $26.20(7.03)$ & 0.66 & 24.89 & 27.51 \\
Bowden \& & $26.41(11.24)$ & 1.06 & 24.31 & 28.50 \\
$\begin{array}{l}\text { Jung-Beeman } \\
\text { Both }\end{array}$ & $52.64(16.16)$ & 1.52 & 49.62 & 55.65 \\
\hline
\end{tabular}

order to native speakers in an online study. Accuracy and response times for solving the items were recorded. The purpose of the study was to check whether: (i) correlations between performance indicators hold between comRAT-G and Bowden \& Jung-Beeman items, thus showing validity of comRAT-G items and (ii) whether comRAT-G queries are a reliable tool, as measured by Cronbach's alpha, compared to Bowden \& Jung-Beeman items.

\section{Participants}

A total of 113 native English speakers, 72 female and 41 male, were recruited at University of Pittsburgh and on Crowdflower and volunteered to answer our study, which was set up online. Participants had a wide range of ages, education levels and self-rated their creativity on a wide set of levels, as shown in Table 2.

\section{Materials}

Fifty compound RAT queries were randomly selected from the items produced by comRAT-G. Another 50 queries were randomly selected from the query set of Bowden \& Jung-Beeman. The comRAT-G queries can be found in the Appendix. From Bowden and Jung-Beeman (2003) we used queries $5,6,11,15,17,20,21,22,24,26,28,29,30,37$, $38,40,45,46,50,51,53,58,62,65,68,71,72,74,76,79$, $82,84,87,90,95,96,99,106,110,111,114,116,122,124$, 130, 131, 133, 136, 139 and 144.

Table 4 Descriptive statistics on response times (RT) in seconds for queries solved, $n=112$

\begin{tabular}{lllll}
\hline & $\begin{array}{l}\text { Mean RT in } \\
\text { seconds }(S D)\end{array}$ & $\begin{array}{l}\text { Std. } \\
\text { Error }\end{array}$ & $\begin{array}{l}95 \% \text { Conf. } \\
\text { L.B. }\end{array}$ & $\begin{array}{l}\text { interval } \\
\text { U.B }\end{array}$ \\
\hline comRAT-G & $14.52(9.89)$ & 0.93 & 12.67 & 16.38 \\
Bowden \& & $16.56(12.84)$ & 1.21 & 14.15 & 18.97 \\
Jung-Beeman & & & & \\
\hline
\end{tabular}


Table 5 Descriptive statistics on number of participants solving per query (of 113), and mean time spent per query (whether a correct answer was given or not)

\begin{tabular}{|c|c|c|c|c|}
\hline & $\begin{array}{l}\text { Mean no. of } \\
\text { participants solving }(S D)\end{array}$ & $\begin{array}{l}\text { Std. } \\
\text { Error }\end{array}$ & $\begin{array}{l}95 \% \text { Conf. } \\
\text { L.B. }\end{array}$ & $\begin{array}{l}\text { interval } \\
\text { U.B }\end{array}$ \\
\hline Solved comRAT-G & $59.28(33.63)$ & 4.76 & 49.72 & 68.84 \\
\hline Solved Bowden \& Jung-Beeman & $59.92(21.18)$ & 3.0 & 53.9 & 65.94 \\
\hline \multirow[t]{2}{*}{ Solved both } & $59.6(27.96)$ & 2.8 & 54.05 & 65.15 \\
\hline & $\begin{array}{l}\text { Mean time } \\
\text { spent solving }\end{array}$ & $\begin{array}{l}\text { Std. } \\
\text { Error }\end{array}$ & $\begin{array}{l}95 \% \text { Conf. } \\
\text { L.B. }\end{array}$ & $\begin{array}{l}\text { interval } \\
\text { U.B }\end{array}$ \\
\hline Time comRAT-G & $21.9(10.3)$ & 1.46 & 18.97 & 24.83 \\
\hline Time Bowden \& Jung-Beeman & $23.12(7.1)$ & 1.00 & 21.11 & 25.14 \\
\hline Time both & $22.51(8.82)$ & 0.88 & 20.76 & 24.26 \\
\hline
\end{tabular}

\section{Procedure}

The task was explained with two query examples. Then, five training queries were presented. These queries were taken from Bowden \& Jung-Beeman items and did not overlap with our random selection of 50 items. After the participants attempted to solve the training queries, feedback including the correct answer was presented. Then, the 100 queries (50 from Bowden \& Jung-Beeman, 50 from comRAT-G) were presented in random order.

\section{Results}

The dependent variables were (i) accuracy, measured as the number of correct responses for each participant in comRAT-G and Bowden \& Jung-Beeman queries, and (ii) response times, measured as the number of seconds each participant spent on answering each comRAT-G and Bowden \& Jung-Beeman query.

As Table 3 shows, the mean accuracy was 26.20, standard deviation $(S D)=7.03)$ problems correctly solved $(52.4 \%)$ for comRAT-G items and $26.41(S D=11.24)$ problems correctly solved $(52.82 \%)$ for Bowden \& Jung-Beeman items. The mean response times for correct solutions $(n=$ $\left.112^{6}\right)$ was $14.52 \mathrm{~s}(S D=9.89)$ for comRAT-G items and $16.56 \mathrm{~s}$ ( $S D=12.84)$ for Bowden \& Jung-Beeman items, as shown in Table 4.

As shown in Table 5, the mean number of participants solving each comRAT-G query was 59.28, and the mean number of participants solving each Bowden \&

\footnotetext{
${ }^{6} \mathrm{We}$ removed one outlier that spent $177 \mathrm{~min}$ on the task, as this indicated solving the RAT was not their main focus.
}

Jung-Beeman query was 59.92. The mean time spent per comRAT-G query, independent of whether it was solved or not, was $21.9 \mathrm{~s}$, while the mean time spent per each Bowden \& Jung-Beeman query was 23.12 .

Accuracy and response times per query for the comRAT$\mathrm{G}$ dataset are shown in the Appendix.
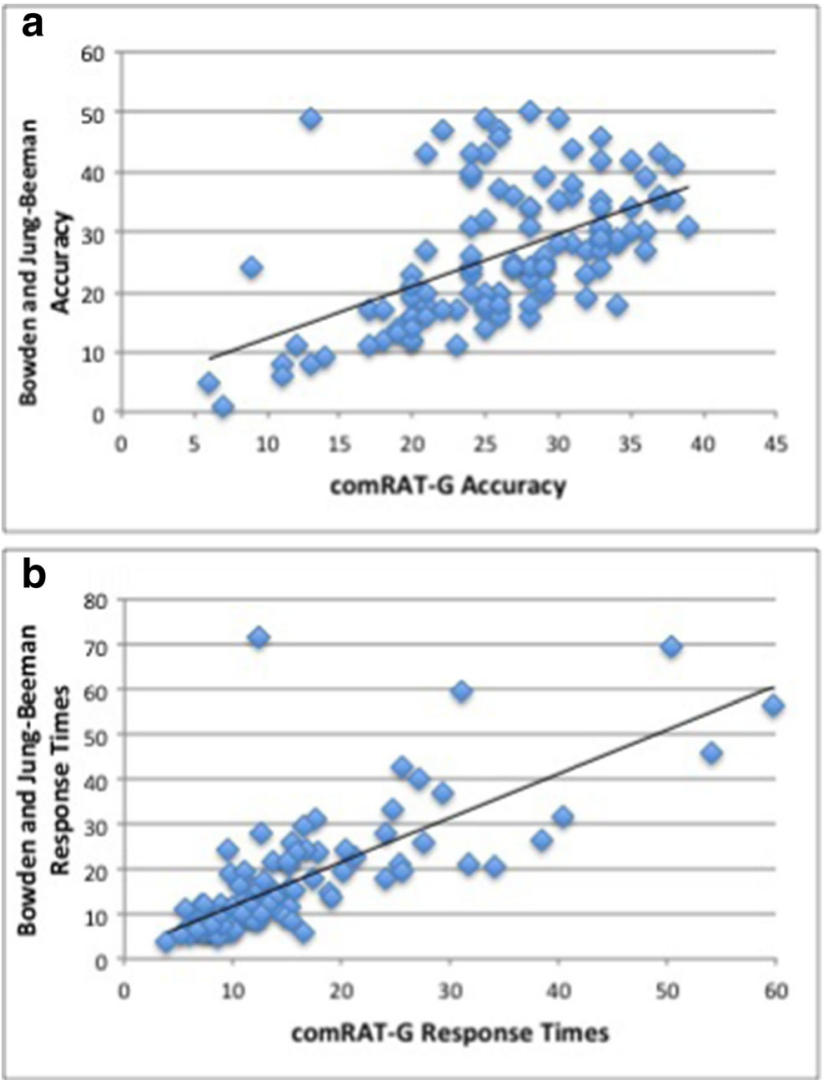

Fig. 2 Correlations on $\mathbf{a}$ accuracy and $\mathbf{b}$ response times 
Accuracy showed an average significant correlation between the comRAT-G and the Bowden \& Jung-Beeman datasets of $r=0.54, p<0.0001$ (Fig. 2a). Response times showed a highly significant large correlation between the two datasets of $r=0.75, p<0.0001$ (Fig. 2b). Note that response times were calculated only for correct answers.

We then measured the scale reliability of the two datasets (comRAT-G items and Bowden \& Jung-Beeman items) using Cronbach's alpha as an internal consistency measure. As Table 6 shows, Cronbach's alpha on accuracy was 0.851 for comRAT-G items, 0.932 for Bowden \& Jung-Beeman items and 0.936 for both sets of queries taken together. Cronbach's alpha on response times (on both correct and incorrect answers) was 0.991 for comRAT-G items, 0.99 for Bowden \& Jung-Beeman items and 0.995 for both set of queries taken together.

As a final point, we checked to see whether the accuracy and response times data we obtained on the Bowden \& Jung-Beeman dataset with our participants correlated with that obtained by Bowden \& Jung-Beeman. As shown in Table 7, all accuracy measures and all but one response times measures correlated significantly.

\section{Discussion}

The descriptive data is similar between the comRAT-G and Bowden \& Jung-Beeman's set of queries, on both mean accuracy and mean response times. The average and high correlations obtained between the performance of the participants on the comRAT-G and Bowden \& Jung-Beeman sets of items on both accuracy and response times show the validity of the comRAT-G dataset, pointing to the fact that we are measuring the same skill with comRAT-G as with Bowden \& Jung-Beeman sets. The high Cronbach's alpha internal consistency scores, which remain the same or increase when putting the two item sets together, show that both sets are highly reliable, and consistent with each other. Thus the comRAT-G data are in all crucial respects similar to the established query set.

Table 6 Cronbach's alpha internal consistency measures

\begin{tabular}{llll}
\hline Set of queries & $\begin{array}{l}\text { Performance } \\
\text { measure }\end{array}$ & $n$ & $\begin{array}{l}\text { Cronbach's } \\
\text { Alpha }\end{array}$ \\
\hline comRAT-G & Accuracy & 113 & 0.851 \\
Bowden \& Jung-Beeman & Accuracy & 113 & 0.932 \\
Both & Accuracy & 113 & 0.936 \\
comRAT-G & Response times & 112 & 0.991 \\
Bowden \& Jung-Beeman & Response times & 112 & 0.99 \\
Both & Response times & 112 & 0.995 \\
\hline
\end{tabular}

Table 7 Correlation of performance on the Bowden \& Jung-Beeman queries, between our participants and Bowden \& Jung-Beeman's participants

\begin{tabular}{llll}
\hline Measure type & $\begin{array}{l}\text { Bowden \& } \\
\text { Jung-Beeman } \\
\text { measure }\end{array}$ & Our measure & Correlation \\
\hline Accuracy & Accuracy 2s & Accuracy & $r=0.66, p<0.0001$ \\
& Accuracy 7s & Accuracy & $r=0.76, p<0.0001$ \\
& Accuracy 15s & Accuracy & $r=0.78, p<0.0001$ \\
Response & Accuracy 30s & Accuracy & $r=0.81, p<0.0001$ \\
times & RT 7s & RT & $r=0.45, p<0.0001$ \\
& RT 15s & RT & $r=0.15,-$ \\
& RT 30s & RT & $r=0.72, p<0.0001$ \\
& & &
\end{tabular}

\section{The Repository}

In the following, the RAT queries generated and the data which accompanies them will be explained as a function of data items (columns), ability to search for and order items and some examples of possible empirical research using this data. Table 8 shows a sample of the generated queries data and their form.

The generated compound RAT queries can thus be ordered in the following ways:

(1) Alphabetically by the first, second and third word (on $w_{a}, w_{b}$ and $w_{c}$ ). This ability to search for alphabetically ordered RAT queries allows empirical research keeping the first letter or the entire query word (or more than one word) constant. This can be used in various forms, at its extension allowing for keeping the entire query constant and checking for different possible answers.

(2) By the answer $\left(w_{a}\right)$. This can allow for comparisons of query difficulty in which the query terms differ, and the answer is kept constant. ${ }^{7}$ Thus for the queries a) HEALTH, CHILD and CENTER and b) INSURANCE, HAIR and CHILD, the answer is the same - CARE, and so is one of the given terms, CHILD. However, the likelihood of reaching this answer is not the same. Keeping the answer the same can help check upon the influence the different terms and their frequency have on the performance.

(3) By frequency of the favorable cases $\left(f r\left(w_{a}, w_{a n s}\right)\right.$, $\left.f r\left(w_{b}, w_{a n s}\right), f r\left(w_{c}, w_{a n s}\right)\right)$.

\footnotetext{
${ }^{7}$ This can also allow for keeping just the initial part of the answer constant, and checking for phonetico-syntactical rather than semantical influences in answer difference.
} 
Table 8 An example of generated queries, organized by $w_{\text {ans }}$ which is ability

\begin{tabular}{lllllllllllllll}
\hline$w_{a}$ & \multirow{2}{*}{$w_{b}$} & \multirow{2}{*}{$w_{c}$} & $\begin{array}{l}f r\left(w_{\text {ans }},\right. \\
\left.w_{a}\right)\end{array}$ & $\begin{array}{l}f r\left(w_{\text {ans }},\right. \\
\left.w_{b}\right)\end{array}$ & $\begin{array}{l}f r\left(w_{\text {ans }},\right. \\
\left.w_{c}\right)\end{array}$ & $\begin{array}{l}f r \\
\left(w_{a}\right)\end{array}$ & $\begin{array}{l}f r \\
\left(w_{b}\right)\end{array}$ & $\begin{array}{l}f r \\
\left(w_{c}\right)\end{array}$ & $\begin{array}{l}P\left(w_{\text {ans }}\right. \\
\left.\mid w_{a}\right)\end{array}$ & $\begin{array}{l}P\left(w_{\text {ans }}\right. \\
\left.\mid w_{b}\right)\end{array}$ & $\begin{array}{l}P\left(w_{\text {ans }}\right. \\
\left.\mid w_{c}\right)\end{array}$ & $P\left(w_{\text {ans }}\right)$ & $w_{\text {ans }}$ \\
\hline coping & language & leadership & 88 & 58 & 76 & 1237 & 6405 & 4556 & 0.0711 & 0.0091 & 0.0167 & 0.0323 & ability \\
coping & language & problem-solving & 88 & 58 & 50 & 1237 & 6405 & 606 & 0.0711 & 0.0091 & 0.0825 & 0.0542 & ability \\
coping & language & reading & 88 & 58 & 65 & 1237 & 6405 & 4292 & 0.0711 & 0.0091 & 0.0151 & 0.0318 & ability \\
coping & language & reasoning & 88 & 58 & 37 & 1237 & 6405 & 121 & 0.0711 & 0.0091 & 0.3058 & 0.1287 & ability \\
coping & language & student & 88 & 58 & 35 & 1237 & 6405 & 26153 & 0.0711 & 0.0091 & 0.0013 & 0.0272 & ability \\
coping & language & thinking & 88 & 58 & 34 & 1237 & 6405 & 1239 & 0.0711 & 0.0091 & 0.0274 & 0.0359 & ability \\
coping & language & writing & 88 & 58 & 29 & 1237 & 6405 & 2515 & 0.0711 & 0.0091 & 0.0115 & 0.0306 & ability \\
\hline
\end{tabular}

(4) By the (sum) frequency of the given words $\left(f r\left(w_{a}\right)\right.$, $\left.f r\left(w_{b}\right), f r\left(w_{c}\right)\right)$. This allows for the study of frequency based influence separately from probability. Empirical exploration of whether keeping the frequency constant throughout the words (query or answer) among different queries has an impact on answer performance, and the function and interinfluences between frequency and answer performance should thus be possible.

(5) By the probability of the answer to be found. Multiple queries with similar probabilities can thus be analyzed, and queries from different probability classes can be analyzed (low probability, medium probability, high probability) together with their influence on human performance.

The frequency of the various query words with the answer, and the frequency of the query words occurring in other combinations has been provided here separately, as the probability of finding the answer has been calculated here taking the influence from the three words to be equal. It might be the case that the first two words have a higher influence (see Olteţeanu, 2014), and showing frequency explicitly for each of the query words allows the study of order effects.

An interface permitting access to the queries constructed by comRAT-G can be found here: http://creacogcomp.com/ comRAT-G.html.

\section{Conclusions and future work}

An ample set of about 17 million queries was generated using a variant of a computational RAT solver - comRAT-G. This set of queries aims to fill a gap in the area of providing normative frequency-based data and an ampler set of stimuli for cognitive and computational creativity research. Frequency and frequency-based probability of finding the answer have been computed for all the generated queries and are provided with this data. The contributed repository allows further control over variables when testing for the influence of frequency, keeping words constant and word order in Remote Associates queries.

The entire list of queries or a subset thereof can be obtained by contacting the authors. As future work, we will aim to make the following contributions:

(i) Improve the online interface with more search and selection features, for easy access.

(ii) Generate an updated version of this repository by also parsing compound nouns from the corpus automatically, and offer queries based on compound nouns as another controllable variable. The motivation for this is that items of the form $\left(w_{a n s}, w_{q}\right)$ which are parsed from compound nouns might be associated more tightly than items which have 2grams as a point of origin.

(iii) Generate a version of the repository which includes queries made of other parts of speech than nouns alone.

(iv) Offer the ability to collapse plural and singular forms to modelers.

(v) Add free association norms data to the query-answer pairs, if available.

(vi) Enable control of query and answer word length.

(vii) Enable control over semantic domain of words.

(viii) Rate a part of these queries for interestingness and hardness, in order to further refine the generating algorithm.

Acknowledgements Ana-Maria Olteţeanu gratefully acknowledges the support of the Deutsche Forschungsgemeinschaft (DFG) for the Creative Cognitive Systems (CreaCogs) project. $^{8}$ The support offered by the RISE DAAD program is acknowledged by Ana-Maria Olteţeanu and Jonathan B. Dyer. We thank Thansuda Kraisangka from Mahidol University for helping us create the interface to the generated test items.

\footnotetext{
${ }^{8}$ http://creacogcomp.com
} 


\section{Appendix: Accuracy and response times per query ${ }^{9}$}

\begin{tabular}{|c|c|c|c|c|c|c|c|c|c|c|c|c|c|c|c|c|}
\hline No & $w_{a}$ & $w_{b}$ & $w_{c}$ & $\begin{array}{l}\left(w_{\text {ans }},\right. \\
\left.w_{a}\right)\end{array}$ & $\begin{array}{l}\left(w_{\text {ans }},\right. \\
\left.w_{b}\right)\end{array}$ & $\begin{array}{l}\left(w_{\text {ans }},\right. \\
\left.w_{c}\right)\end{array}$ & $\begin{array}{l}f r \\
\left(w_{a}\right)\end{array}$ & $\begin{array}{l}f r \\
\left(w_{b}\right)\end{array}$ & $\begin{array}{l}f r \\
\left(w_{c}\right)\end{array}$ & $\begin{array}{l}P\left(w_{\text {ans }}\right. \\
\left.\mid w_{a}\right)\end{array}$ & $\begin{array}{l}P\left(w_{\text {ans }}\right. \\
\left.\mid w_{b}\right)\end{array}$ & $\begin{array}{l}P\left(w_{\text {ans }}\right. \\
\left.\mid w_{c}\right)\end{array}$ & $P\left(w_{\text {ans }}\right)$ & $w_{\text {ans }}$ & $\begin{array}{l}\text { Solved } \\
(\mathrm{n}=113)\end{array}$ & RT (SD) \\
\hline 1 & box & panes & shades & 86 & 57 & 79 & 8815 & 123 & 108 & 0.0098 & 0.4634 & 0.7315 & 0.4016 & window & 79 & 13.39 (18.16) \\
\hline 2 & bicycle & pawn & photo & 45 & 104 & 35 & 323 & 149 & 2936 & 0.1393 & 0.6980 & 0.0119 & 0.2831 & shop & 94 & $14.1(14.72)$ \\
\hline 3 & penalty & suit & toll & 5195 & 44 & 1045 & 6127 & 3016 & 1471 & 0.8479 & 0.0146 & 0.7104 & 0.5243 & death & 8 & $11.85(8.74)$ \\
\hline 4 & paddle & roulette & steering & 27 & 57 & 1809 & 79 & 95 & 2469 & 0.3418 & 0.6000 & 0.7327 & 0.5582 & wheel & 91 & $12.1(10.98)$ \\
\hline 5 & gala & invitation & table & 55 & 29 & 1120 & 130 & 90 & 16357 & 0.4231 & 0.3222 & 0.0685 & 0.2713 & dinner & 15 & $27.51(29.17)$ \\
\hline 6 & cellars & regions & shop & 29 & 27 & 61 & 55 & 231 & 7480 & 0.5273 & 0.1169 & 0.0082 & & wine & & 18.24 (26.99) \\
\hline 7 & closing & departure & lunch & 84 & 40 & 75 & 195 & 107 & 2903 & 0.4308 & 0.3738 & 0.0258 & 0.2768 & date & & $14.92(14.45)$ \\
\hline 8 & cedar & fig & bark & 40 & 122 & 126 & 172 & 351 & 342 & 0.2326 & 0.3476 & 0.3684 & 0.3162 & tree & 5 & 13.98) \\
\hline 9 & musicians & protests & shoes & 42 & 130 & 30 & 418 & 195 & 1814 & 0.1005 & 0.6667 & 0.0165 & 0.2612 & street & 16 & 46.92) \\
\hline 10 & gain & prosperity & wealth & 50 & 38 & 120 & 1232 & 77 & 797 & 0.0406 & 0.4935 & 0.1506 & 0.2282 & material & 1 & $(-)$ \\
\hline 11 & ocean & tile & level & 504 & 327 & 72 & 2075 & 634 & 15700 & 0.2429 & 0.5158 & 0.0046 & 0.2544 & floor & 52 & 17.76) \\
\hline 12 & pep & pillow & host & 263 & 70 & 26 & 558 & 203 & 3466 & 0.4713 & 0.3448 & 0.0075 & 0.2745 & talk & & \\
\hline 13 & bait & hatch & tanks & 27 & 37 & 54 & 89 & 77 & & & & & & fish & & \\
\hline 14 & pounding & attacks & defect & 62 & 1204 & 54 & 710 & 3394 & 167 & 0.0873 & 0.3547 & 0.3234 & 0.2551 & heart & 7 & .13) \\
\hline 15 & harmony & saturation & schemes & 36 & 33 & 107 & 75 & 263 & 274 & 0.4800 & 0.1255 & 0.3905 & & color & & \\
\hline 16 & enemies & scoring & word & 56 & 25 & 43 & 89 & 606 & 2799 & 0.6292 & 0.0413 & 0.0154 & 0.2286 & list & & .71) \\
\hline 17 & dealers & industry & mechanic & 104 & 1232 & 167 & 2128 & 28295 & 212 & 0.0489 & 0.0435 & 0.7877 & 0.2934 & auto & 100 & \\
\hline 18 & complaints & fraud & tastes & 136 & 52 & 36 & 414 & 1845 & 73 & 0.3285 & 0.0282 & 0.4932 & 0.2833 & consumer & 15 & $5.22)$ \\
\hline 19 & clippings & columns & story & 23 & 68 & 129 & 557 & 244 & 9643 & 0.4219 & 0.2787 & 0.0134 & 0.2 & new & 96 & 54) \\
\hline 20 & cartoon & actors & flaws & 19 & 69 & 68 & 565 & 30 & 13 & 0.3487 & 0.2248 & 0.50 & & & 66 & $.57)$ \\
\hline 21 & banker & plan & strategies & 640 & 53 & 63 & 672 & & & & & & & investn & 29 & \\
\hline 22 & cup & crate & powd & 415 & 62 & 55 & 13087 & 93 & 3222 & 0.0317 & & 0.0171 & & milk & 5 & .42) \\
\hline 23 & checking & escrow & defici & 33 & 110 & 34 & 405 & 134 & 976 & 0.8321 & 0.8209 & 0.03 & & accoun & 82 & \\
\hline 24 & holiday & wind & mall & 251 & 42 & 867 & 2469 & 7884 & 1306 & 0.1017 & 0.0053 & 0.6639 & 0.2 & shol & 75 & 97) \\
\hline 25 & bikes & climber & peak & 328 & 73 & 63 & 479 & 184 & 809 & 0.6848 & 0.3967 & 0.0779 & 0.3 & mount & 100 & \\
\hline 26 & climbers & format & song & 67 & 96 & 56 & 129 & 1437 & 2305 & 0.5194 & 0.0668 & 0.0243 & 0.2035 & rock & 40 & .71) \\
\hline 27 & health & track & labels & 35 & 1609 & 249 & 79202 & 4132 & 741 & 0.0004 & 0.3894 & 0.3360 & 0.2419 & record & 6 & \\
\hline 28 & baseball & neighb & ranger & 45 & 59 & 217 & 9730 & & & & & & & park & & \\
\hline 29 & cold & dancing & hockey & 88 & 41 & 341 & 222 & 465 & 2406 & 0.3964 & 0.0882 & 0.1417 & 0.2088 & & 89 & \\
\hline 30 & & & & 33 & 33 & & & & & & & & & & 75 & \\
\hline 31 & clip & footage & games & 306 & 128 & 1820 & 797 & 825 & 8054 & 0.3839 & 0.1552 & 0.2260 & 0.2 & video & 41 & \\
\hline 32 & creature & foam & snakes & 70 & 33 & 47 & 212 & 491 & 119 & 0.3302 & 0.0672 & 0.3950 & 0.2641 & sea & 57 & $\begin{array}{l}29.65) / \\
15.98)\end{array}$ \\
\hline 33 & embar & fire & & 572 & 53 & 75 & & & & & & & & & 6 & \\
\hline 34 & florist & hangers & whisk & 30 & 28 & 136 & 60 & 88 & 160 & 0.5000 & 0.3182 & 0.8500 & 0.5561 & wire & 24 & $\begin{array}{l}9) / \\
.82)\end{array}$ \\
\hline 35 & & & & 43 & & & 10 & & & & & & & & & \\
\hline 36 & breeze & urchi & voyage & 97 & 173 & 53 & 210 & 202 & 108 & 0.4619 & 0.8564 & 0.4907 & 0.6 & sea & 92 & \\
\hline 37 & chores & incomes & pets & 302 & 132 & 51 & 351 & 252 & 113 & 0.8604 & 0.5238 & 0.4513 & 0.6118 & household & 76 & 23.58 \\
\hline 38 & blades & coaster & skates & 29 & 1026 & 97 & 1113 & 1125 & 155 & 0.0261 & 0.9120 & 0.6258 & 0.5213 & roller & 98 & $8.56(8.15)$ \\
\hline 39 & blouse & handkerchief & ribbon & 162 & 55 & 26 & 230 & 84 & & 0.7043 & & 0.1677 & & & 43 & 11.05) \\
\hline 40 & math & obstacle & refresher & 30 & 340 & 95 & 2484 & 389 & 124 & 0.0121 & 0.8740 & 0.7661 & 0.5507 & cour & 81 & 12.84) \\
\hline 41 & lot & spaces & ticket & 8264 & 288 & 112 & & 472 & & 0.9357 & & & & & 94 & \\
\hline 42 & juice & tart & zest & 3506 & 44 & 601 & 9568 & 107 & 758 & & & 0.7929 & & lemon & 70 & \\
\hline 43 & deterrent & ripple & snowball & 110 & 320 & 40 & 135 & 434 & 116 & 0.8148 & 0.7373 & 0.3448 & 0.6323 & effect & 59 & $\begin{array}{l}24.46(41.53) / \\
20.46(28.45)\end{array}$ \\
\hline 44 & brown & cookies & & 69 & 37 & & 97 & 552 & 235 & 0.7113 & 0.0670 & 0.8638 & 0.5474 & chocolate & 99 & $16.57(20.7)$ \\
\hline 45 & nest & wash & yolk & 382 & 64 & 346 & 657 & 553 & 416 & 0.5814 & 0.1157 & 0.8317 & 0.5096 & egg & 99 & 9.85 (7.76) \\
\hline 46 & bolts & bugs & storm & 131 & 79 & 89 & 156 & 104 & 2802 & 0.8397 & 0.7596 & 0.0318 & 0.5437 & lightning & 61 & $\begin{array}{l}29.38(104.9) / \\
16.08(14.72)\end{array}$ \\
\hline 47 & nucleus & phones & & 31 & & 31 & 57 & & & & & & & & 75 & \\
\hline 48 & concern & discrepancy & lifting & 33 & 38 & 197 & 268 & 64 & 226 & 0.1231 & 0.5938 & 0.8717 & 0.5295 & weight & 18 & $\begin{array}{l}37.1(57.56) / \\
17.61(10.47)\end{array}$ \\
\hline 49 & apnea & & & 332 & 197 & 50 & 301 & & 1045 & 0.9197 & 0.6396 & 0.0287 & & sleep & 98 & $6.89(4.9)$ \\
\hline 50 & jump & resorts & slope & 71 & 165 & 81 & 790 & 189 & 122 & 0.0899 & 0.8730 & 0.6639 & 0.5423 & ski & 95 & $7.37(7.12)$ \\
\hline
\end{tabular}

${ }^{9}$ Response times and SD for queries 29, 32, 34, 43, 46 and 48 have also been shown with corrections for outliers. All the data analyses have been done without these corrections. 


\section{References}

Ansburg, P. I., \& Hill, K. (2003). Creative and analytic thinkers differ in their use of attentional resources. Personality and Individual Differences, 34(7), 1141-1152.

Arden, R., Chavez, R. S., Grazioplene, R., \& Jung, R. E. (2010). Neuroimaging creativity: a psychometric view. Behavioural Brain Research, 214(2), 143-156.

Baba, Y. (1982). An analysis of creativity by means of the remote associates test for adult revised in Japanese (JARAT FORM a). The Japanese Journal of Psychology, 52(6), 330-336.

Bowden, E. M., \& Jung-Beeman, M. (2003). Normative data for 144 compound remote associate problems. Behavior Research Methods, Instruments, and Computers, 35(4), 634-639.

Bringsjord, S. (2011). Psychometric artificial intelligence. Journal of Experimental \& Theoretical Artificial Intelligence, 23(3), 271277.

Cai, D. J., Mednick, S. A., Harrison, E. M., Kanady, J. C., \& Mednick, S. C. (2009). REM, not incubation, improves creativity by priming associative networks. Proceedings of the National Academy of Sciences, 106(25), 10130-10134.

Chermahini, S. A., Hickendorff, M., \& Hommel, B. (2012). Development and validity of a Dutch version of the remote associates task: An item-response theory approach. Thinking Skills and Creativity, 7(3), 177-186.

Duncker, K. (1945). On problem solving. Psychological Monographs, 58(5), i-113.

Fodor, E. (1999). Subclinical inclination toward manic-depression and creative performance on the remote associates test. Personality and Individual Differences, 27(6), 1273-1283.

Guilford, J. P. (1967). The nature of human intelligence. McGrawHill.

Kim, K. H. (2006). Can we trust creativity tests? A review of the Torrance tests of creative thinking (TCT). Creativity Research Journal, 18(1), 3-14.

Maier, N. R. (1931). Reasoning in humans. ii. The solution of a problem and its appearance in consciousness. Journal of Comparative Psychology, 12(2), 181.

Mednick, S. A., \& Mednick, M. (1971). Remote associates test: Examiner's manual. Houghton Mifflin.

Olteţeanu, A.-M. (2014). Two general classes in creative problemsolving? An account based on the cognitive processes involved in the problem structure - representation structure relationship. In Besold, T., Kühnberger, K. U., Schorlemmer, M., \& Smaill, A. (Eds.) Proceedings of the international conference on computational creativity, Vol. 01-2014: Osnabrück.

Olteţeanu, A.-M. (2016). From simple machines to eureka in four not-so-easy steps. Towards creative visuospatial intelligence. In Müller, V. (Ed.) Fundamental issues of artificial intelligence. https://doi.org/10.1007/978-3-319-26485-1_11, (Vol. 376 pp. 159_ 180): Springer.

Olteţeanu, A.-M., \& Falomir, Z. (2015). ComRAT-c - a computational compound remote associates test solver based on language data and its comparison to human performance. Pattern Recognition Letters, 67, 81-90. https://doi.org/10.1016/j.patrec.2015.05. 015 .

Olteţeanu, A.-M., \& Falomir, Z. (2016). Object replacement and object composition in a creative cognitive system. Towards a computational solver of the alternative uses test. Cognitive Systems Research, 39, 15-32. https://doi.org/10.1016/j.cogsys.2015.12. 011.

Olteţeanu, A.-M., \& Schultheis, H. (in press). What determines creative association? revealing two factors which separately influence the creative process when solving the remote associates test. The Journal of Creative Behaviour. https://doi.org/10.1002/jocb.177.

Olteţeanu, A.-M., Gautam, B., \& Falomir, Z. (2015). Towards a visual remote associates test and its computational solver. In Proceedings of the third international workshop on artificial intelligence and cognition 2015, (Vol. 1510 pp. 19-28): CEUR-Ws.

Olteţeanu, A.-M., Falomir, Z., \& Freksa, C. (in press). Artificial cognitive systems that can answer human creativity tests: an approach and two case studies. IEEE Transactions on Cognitive and Developmental Systems. https://doi.org/10.1109/TCDS.2016.2629622.

Qiu, J., Li, H., Yang, D., Luo, Y., Li, Y., Wu, Z., \& Zhang, Q. (2008). The neural basis of insight problem solving: an event-related potential study. Brain and Cognition, 68(1), 100-106.

Saugstad, P., \& Raaheim, K. (1957). Problem-solving and availability of functions. Acta Psychologica, 13, 263-278.

Schooler, J. W., \& Melcher, J. (1995). The ineffability of insight. In Ward, T., \& Finke, R. (Eds.) The creative cognition approach (pp. 249-268). Cambridge, MA: The MIT Press.

Sio, U. N., \& Rudowicz, E. (2007). The role of an incubation period in creative problem solving. Creativity Research Journal, 19(2-3), 307-318.

Sitton, S. C., \& Pierce, E. R. (2004). Synesthesia, creativity and puns 1. Psychological Reports, 95(2), 577-580.

Storm, B. C., Angello, G., \& Bjork, E. L. (2011). Thinking can cause forgetting: memory dynamics in creative problem solving. Journal of Experimental Psychology: Learning, Memory, and Cognition, $37(5), 1287$.

Tucker, A. (2006). Applied combinatorics. Wiley.

Wallach, M. A., \& Kogan, N. (1965). Modes of thinking in young children: A study of the creativity-intelligence distinction. Holt, Rinehart \& Winston.

Ward, J., Thompson-Lake, D., Ely, R., \& Kaminski, F. (2008). Synaesthesia, creativity and art: What is the link? British Journal of Psychology, 99(1), 127-141.

Whitt, J. K., \& Prentice, N. M. (1977). Cognitive processes in the development of children's enjoyment and comprehension of joking riddles. Developmental Psychology, 13(2), 129.

Wiley, J. (1998). Expertise as mental set: The effects of domain knowledge in creative problem solving. Memory \& Cognition, 26(4), 716-730.

Worthen, B. R., \& Clark, P. M. (1971). Toward an improved measure of remote associational ability. Journal of Educational Measurement, 8(2), 113-123. 